\section{Cervical Spine Immobilization Device for Emergency Response}

\author{
Alperen Degirmenci ${ }^{1}$, Benjamin Goldberg ${ }^{1}$, Lukas \\ Bielskis $^{1}$, Shanna Wiggins ${ }^{1}$, Panagiotis \\ Polygerinos $^{1,2}$, Dónal Holland ${ }^{1,3}$, Dr. Sophia Dyer ${ }^{4}$, \\ Conor J. Walsh ${ }^{1,2}$ \\ ${ }^{1}$ School of Engineering and Applied Sciences \\ ${ }^{2}$ Wyss Institute for Biologically Inspired Engineering \\ Harvard University \\ ${ }^{3}$ Trinity College Dublin, Ireland \\ ${ }^{4}$ Boston EMS, Boston Medical Center
}

\section{Background}

Cervical spine injuries are a significant cause of morbidity and mortality each year in the United States [1]. In motor vehicle accidents requiring the vehicle to be towed, $33 \%$ of passengers will sustain a severe neck injury [2]. In the current standard of care, a cervical collar (c-collar) is used to stabilize a patient's cervical spine and head in a neutral position. However, in many cases, additional stabilization is required to properly constrain head and neck movement. These supplementary constraints include strapping the patient to a rigid plastic backboard and placing foam blocks on either side of the head. The noncompliant nature of the backboard can create localized pressure points on the patient's back, causing a variety of problems, such as tissue ischemia and ulcers [3]. The pressure exerted by the c-collar on the carotid arteries can lead to raised intracranial pressure [4]. Most importantly, since the c-collar is not fully adjustable to fit all patients, the neck can be overextended, leading to vertebral separation [5].

The emergency response device presented in this paper easily, safely, and comfortably stabilizes the upper cervical spine of a patient while allowing for maximum conformability and versatility to prevent further injuries. Experimental results show that compared to the currently used cervical collars, the new device reduces neck movement by more than $56 \%$ in the three rotational degrees of freedom of the neck.

\section{Methods}

Discussions with clinicians and paramedics helped identify a set of functional requirements, which fall into four categories: 1) stability, 2) adjustability, 3) conformability, and 4) safety. First and foremost, the device must properly immobilize the head. Clinicians have defined 'proper immobilization' as less than 10 degrees of movement in the pitch, roll, and yaw rotational degrees of freedom of the neck [6]. The current c-collars come in four discrete sizes pediatric, small, medium, and large; but a continuously adjustable device that is intuitive and easy to apply is desired. The design should eliminate the problematic pressure points caused by the current c-collars which are at the top and bottom of the neck, on the carotid arteries, or on the back of the head (if the patient is on a rigid backboard). The device should be transparent to medical imaging since most patients with head trauma will immediately be taken to receive MRI or CT scans. Lastly, the device should maximize access to the patient and allow for other emergency medical procedures to

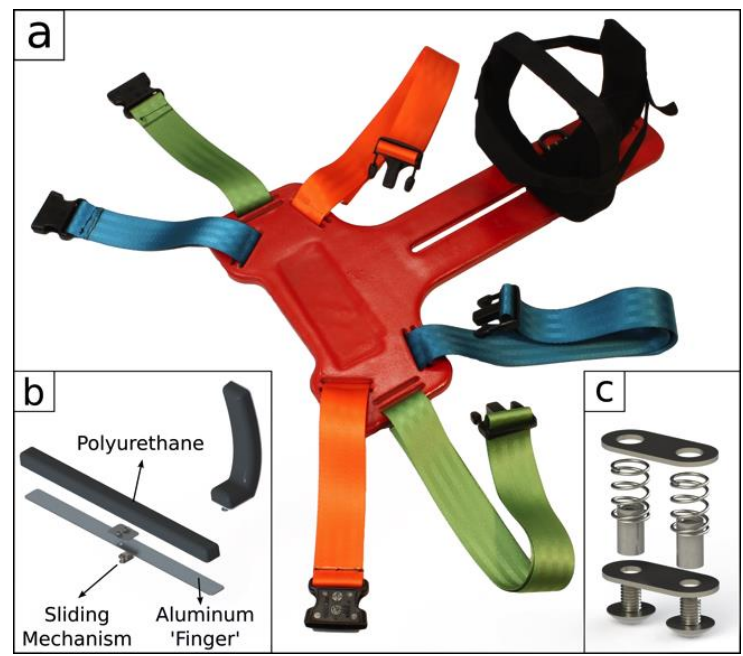

Figure 1: (a) Cervical spine immobilization device; (b) 'finger' elements; (c) sliding mechanism.

be performed. For example, the front of the device should allow access for a surgical airway to be opened.

A literature search, numerous conceptual designs, a Pugh matrix evaluation of design concepts, and many prototype iterations led to the final design shown in Fig. 1a. This device combines a set of straps and height-adjustable head braces to stabilize the head and the cervical spine, thereby securing the patient to a padded, comfortable backplate.

There are two finger-like elements that wrap around and stabilize the head to the backplate (Fig. 1b). The 'fingers' are secured to the patient with a forehead and chin strap. These fingers are made from a flexible aluminum (0.040" 1100 Series Alloy) that is easy to bend into a desired shape and maintain contact with the top and sides of the head. The aluminum plate is coated with a 2" thick layer of foam (opencell Polyurethane) for better conformability and comfort to the patient's head. The bottom of the fingers is coated with Neoprene (1/16", 40A Durometer) and the entire body is then covered in Spandex for comfort, aesthetics, and durability. The forehead strap attaches to the fingers using Velcro to ensure proper immobilization of the head in the pitch direction, preventing the head from slipping forward and out of the device. The chin strap is also attached to the fingers using Velcro and immobilizes the head in the yaw and roll directions by compressing the forehead finger to the patient's head and locking the chin in place.

Each finger is attached to a sliding mechanism, enabling height adjustment (Fig. 1c). This mechanism slides in a channel on the backside of the backplate. Movement of the fingers is allowed when the fingers are pulled away from the

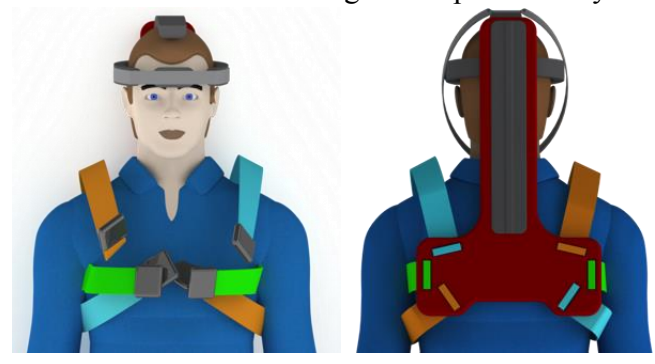

Figure 2: Device applied to a patient. 


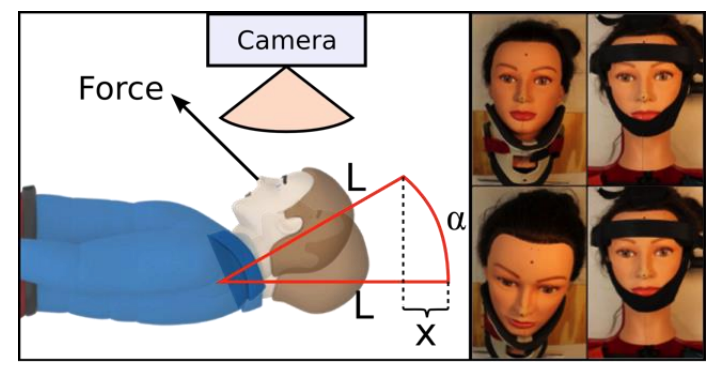

Figure 3: (left) Experimental setup for measuring head deflection in the pitch direction, (right) images showing head analogues in the neutral position (top), and deflected in response to $3 \mathrm{~kg}$ of force (bottom).

backplate. Two springs within the sliding channel compress the fingers to the backboard and prevent the fingers from sliding when at rest.

The backboard of the device consists of a rigid board $\left(1 / 2\right.$ " Delrin $\left.{ }^{\circledR}\right)$ coated with a foam layer ( $1 / 4$ " Vinyl) for patient comfort as well as increased friction with the fingers when they are locked in place. A steel plate placed between these two layers along the channel of the board provides a narrow lip that holds the sliding mechanism inside the channel.

A set of three chest straps anchors the device to the chest, preventing the patient from shifting relative to the backplate. Two of the chest straps cross diagonally across the patient's chest, going over the shoulder on one side and under the shoulder on the other side. The third strap goes horizontally across the chest (Fig. 2). This setup allows for high comfort and adjustability. Such strapping configuration is intentionally redundant and is aimed to ensure patient-specific adaptability for cases in which certain forces need to be avoided. For example, in a situation where the patient maintains a dislocated shoulder, one of the straps can be left detached while still maintaining sufficient cervical spine immobilization.

\section{Results}

The performance of the currently used c-collars was compared to the new device in a head deflection test. For this purpose, a mannequin head was used as an analogue for a human head. To simulate the functionality of the cervical spine, the head was mounted on a ball joint connected to a rigid bar. The same amount of force $(3 \mathrm{~kg}$ in roll and pitch, 2 $\mathrm{kg}$ in yaw) was applied to the mannequin using a spring scale to simulate torques in the roll, pitch, and yaw degrees of

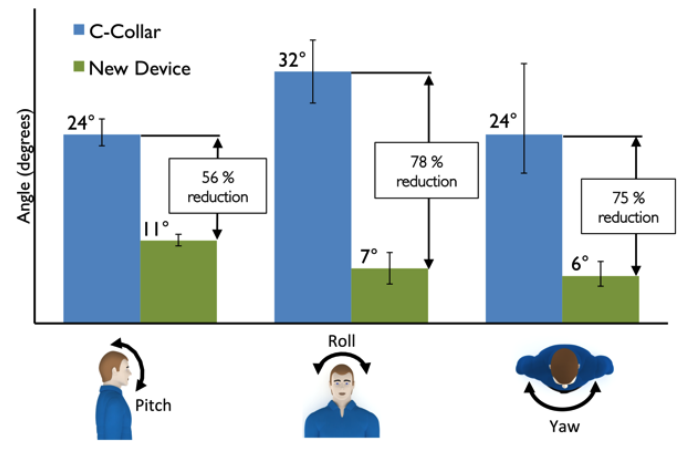

Figure 4: Comparison of the amount of movement allowed with each device. freedom (Fig. 3). Using video tracking (Image-J, NIH), the amount of movement allowed with each of the devices was quantified by tracking two fiducial markers on the mannequin's head, one on the nose, and one on the forehead.

The following equations give the relation between the projection of the change in marker position, $x$, and the angle of head deflection from neutral position, $\alpha, \beta$, and $\gamma$ corresponding to the pitch, roll, and yaw degrees of freedom, respectively, where $L$ is the distance from the center of rotation (ball joint) to each marker, and $R$ is the head radius. $\alpha=\cos ^{-1}\left(\frac{L-x}{L}\right), \beta=\cos ^{-1}\left(1-\frac{x^{2}}{2 L^{2}}\right), \gamma=\sin ^{-1}\left(\frac{x}{R}\right)$

Fig. 4 shows that the new device outperforms the c-collar at stabilizing the head of the mannequin. Compared to the ccollar, the new device decreases the amount of movement allowed by more than $56 \%$ in each degree of freedom, up to $78 \%$ in the roll degree of freedom.

\section{Interpretation}

Experimental results show that the device presented in this paper outperforms the currently used c-collars in immobilizing head and neck movement, thus providing a viable alternative for use in emergency medicine. The device lowers risk of further injury by meeting the "proper immobilization' goal (maximum 10 degrees of movement) set by clinicians and maximizes patient comfort. The new device reduces the cervical spine immobilization time to less than two minutes, allowing emergency personnel to tend to additional urgent medical needs of the patient. The adaptability of fingers to patient-specific anatomy alleviates the problem of overextension of the neck. Future work involves making the device compatible with medical imaging, quantitatively confirming the elimination of pressure points and neck overextension, and improving the intuitiveness and robustness of the design for ease-of-use.

\section{References}

[1] G. Alker Jr, et al., High cervical spine and craniocervical junction injuries in fatal traffic accidents: a radiological study, Orth. Clinics of N. America, vol. 9, pp. 1003, (1978).

[2] D.R. Chandler, et al., Emergency Cervical-Spine Immobilization, Annals of Emergency Medicine, vol. 21, no. 10, pp. 1185-88, (1992).

[3] C. Dehner, et al., Comparison of the relative benefits of 2 versus 10 days of soft cervical collar immobilization after acute whiplash injury, Arch Phys Med Rehabil, vol. 87, 1423-27, (2006).

[4] R.J. Mobbs, et al., Effect of cervical hard collar on intracranial pressure after head injury, ANZ Journal of Surgery, vol. 72, 389-91, (2002).

[5] P. Ben-Galim, et al. Extrication collars can result in abnormal separation between vertebrae in the presence of a dissociative injury, J Trauma, vol 69, no 2, pp 447-50, (2010). [6] Dyer, Sophia. Personal Interview. February 7, 2013.

Acknowledgement: This work was performed in ES 227, a course at the Harvard School of Engineering and Applied Sciences (SEAS) taught by one of the authors (CJW) with two of the authors (DH, PP) acting as support teaching staff. Funding for this work was provided by SEAS, the Wyss Institute for Biologically Inspired Engineering, and a Course and Program Grant from the National Collegiate Inventors and Innovators Alliance. 